\title{
USO DE HOSPEDADORES POR EL MUÉRDAGO ARGENTINO LIGARIA CUNEIFOLIA (LORANTHACEAE) A LO LARGO DE SU DISTRIBUCIÓN GEOGRÁFICA
}

\author{
Host use by the Argentine mistletoe LIGARIA CUNeifolia \\ (LORANTHACEAE) ALONG ITS GEOGRAPHICAL DISTRIBUTION
}

Laboratorio Ecotono, INIBIOMA, CONICET-Universidad Nacional del Comahue. Quintral 1250, 8400, Bariloche, Río Negro, Argentina.

*guillermo.amico@comahueconicet.gob.ar

\section{Citar este artículo} AMICO, G. C. \& R.VIDAL-RUSSELL. 2019. Uso de hospedadores por el muérdago argentino Ligaria cuneifolia (Loranthaceae) a lo largo de su distribución geográfica. Bol. Soc. Argent. Bot. 54: 395-403.

DOI: http://dx.doi. org/10.31055/1851.2372.v54. n3.25364

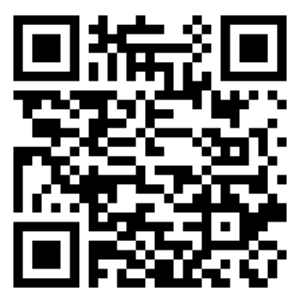

\author{
Guillermo Cesar Amico*(D) y Romina Vidal-Russell(D)
}

\section{RESUMEN}

Introducción y objetivos: Ligaria cuneifolia (Loranthaceae) posee una amplia distribución desde Perú al centro de Argentina y Uruguay. Esta especie habita en tres regiones geográficas en forma disyunta a lo largo de cordillera de los Andes, las Sierras Centrales de Argentina y en el este de Sudamérica, en Uruguay y sur de Brasil. El objetivo de este trabajo fue determinar el rango de hospedadores de Ligaria cuneifolia en toda su distribución geográfica y analizar el uso de hospedadores en cada una de las regiones geográficas (Andes, Centro y Este).

M\&M: Se registró el uso de hospedadores de Ligaria cuneifolia y distribución geográfica en base a ejemplares depositados en herbarios.

Resultados: En total se registraron 35 especies de hospedadores nativos, comprendidas en 22 géneros y 12 familias. Además se registaron 11 especies no nativas en todas las areas. Los géneros de hospedadores con mayor número de registros fueron Schinus, Prosopis, Geoffroea, Larrea y Vachellia.

Conclusiones: El uso de hospedadores no estuvo directamente asociado a las áreas geográficas. La región Andes fue la que presentó una mayor diversidad de especies, géneros y familias de hospedadores.

\section{Palabras clave}

Andes, generalista, hospedadores, muérdagos, parásito.

\section{SUMmaRY}

Background and aims: Ligaria cuneifolia (Loranthaceae) has a wide geographic distribution from Peru to the center of Argentina and Uruguay. It inhabits three disjunct regions, one along the Andes, Sierras Centrales of Argentina and towards the East in Uruguay and south of Brazil. The objective of this study was to determine the host range of Ligaria cuneifolia in all the distribution range; and to analyze host use in each of the geographic regions (Andes, Center and East).

M\&M: hosts use and geographical distribution of Ligaria cuneifolia was recorded on the basis of specimens deposited in herbaria.

Results: A total of 35 native host species were recorded as hosts. In addition, 11 non-native host species were recorded in all area. The genera with higher number of records were Schinus, Prosopis, Geoffroea, Larrea y Vachellia.

Conclusions: Host use was not directly related to geographic area. The Andes region was the one that had the most host diversity at the species, genera and family level.

KEY WORDS

Andes, host, generalist, mistletoe, parasite.

\section{INTRODUCCIÓN}

Loranthaceae, con cerca de 1000 especies, es una de las familias más Recibido: 7 Junio 2019 Aceptado: 29 Julio 2019 Publicado: 30 Septiembre 2019 Editor: Omar Varela (iD grande dentro de los muérdagos (plantas parásitas aéreas) (Mathiasen et al., 2008). Esta familia es considerada de origen Gondwánico y presenta su mayor grado de especiación en zonas subtropicales-tropicales del hemisferio sur (Barlow, 1983; Liu et al., 2018). En América se han descriptos 18 géneros con más de 300 especies (Vidal-Russell \& Nickrent, 
2008; Kuijt \& Hansen, 2015). Solo cinco de los 18 géneros presentes en América tienen pocas especies. Entre estos cinco está Tripodanthus Tiegh. y Ligaria Tiegh con pocas especies, mientras que los restantes son monoespecíficos: Desmaria Tiegh., Gaiadendron G.Don y Notanthera G.Don. A pesar de la gran distribución geográfica y de habitar una gran variedad de ambientes, el género Ligaria sólo posee dos especies (Kuijt, 1990). Ligaria teretiflora (Rizzini) Kuijt, es endémica de Brasil, mientras que L. cuneifolia Tiegh. posee una amplia distribución desde Perú al centro de Argentina y Sur de Brasil. Esta última especie habita en tres regiones geográficas en forma disyunta: los Andes (a lo largo de la cordillera de los Andes, desde Perú a Argentina y Chile; región Andes de aquí en adelante), las Sierras Centrales de Argentina (provincias de Córdoba y San Luis; región Central de aquí en adelante) y el Este del Sur de Sudamérica (Uruguay, Brasil y Mesopotamia Argentina; región Este de aquí en adelante; Fig. 1).

En las regiones Andes y Central se la encuentra por encima de los 700 m.s.n.m., mientras que en la región Este, está en bajas altitudes. Ligaria cuneifolia se encuentra en una gran variedad de ecorregiones. En la región Andes, esta presente en las ecorregiones de Bosque seco boliviano, Matorral chileno, Monte (bajo y alto) y Puna (central seca) según Olson et al. (2001, Fig. 1). En la región Central, se encuentra solo en el Sur de la ecorregión del Chaco Seco. En la región Este, en el Espinal, Sabana uruguaya y Pampas, donde $L$. cuneifolia está asociada a los corredores ribereños.

El rango de hospedadores de Ligaria cuneifolia a lo largo de su amplia y discontinua distribución es poco conocido. Se ha reportado que L. cuneifolia es un muérdago generalista en cuanto al uso de hospedadores, parasitando principalmente a especies de Fabaceae (Abbiatti, 1946). Dentro de cada región geográfica, se desconoce si presenta mayor afinidad al uso de una especie, un género o una familia como hospedador principal. En plantas parásitas, se ha postulado que los hospedadores pueden ejercer presiones de selección divergente y determinar diferentes razas dentro de una especie de muérdago, siendo también un factor importante de diversificación (Norton \& Carpenter, 1998; Lira-Noriega \& Peterson, 2014). El objetivo de este trabajo fue determinar el rango de hospedadores de Ligaria cuneifolia en toda su distribución geográfica. Como así también, analizar el uso de hospedadores de L. cuneifolia a nivel de género y/o familia en cada una de las regiones geográficas (Andes, Centro y Este) con el fin de detectar diferencias en la preferencia de uso de hospedadores a nivel geográfico.

\section{Materiales y Métodos}

Se registró el uso de hospedadores de Ligaria cuneifolia en base a ejemplares depositados en nueve herbarios: BCRU (Bariloche, Argentina), CONC (Concepción, Chile), CTES (Corrientes, Argentina), CORD (Córdoba, Argentina), MA (Madrid, España), MERL (Mendoza, Argentina), MO (Saint Louis, EE. UU.), MVFA (Montevideo, Uruguay) y SI (Buenos Aires, Argentina). Cada ejemplar de herbario fue examinado e identificado a nivel de especie. La especie de hospedador se registró cuando esta información estaba disponible en la etiqueta o si el hospedador se recolectó junto con el muérdago. En este último caso se determinó a nivel de género y si era posible hasta especie. Adicionalmente, se construyó una matriz de datos con la información presente en la etiqueta (hospedador, coleccionista, fecha, localidad, altitud y coordenadas geográficas, cuando estas estaban disponibles). Las muestras duplicadas se excluyeron de la matriz; sin embargo, se incluyeron registros de la misma localidad geográfica. Se sabe que existen sesgos asociados con los datos de herbario, por ejemplo, sesgo de coleccionista (Garcillán \& Ezcurra, 2011); sin embargo, los datos de herbario se pueden usar como una buena aproximación para patrones generales de rango de uso de hospedadores. Este enfoque se ha utilizado en otros estudios de muérdago en diferentes partes del mundo (Downey, 1998; Norton \& De Lange, 1999; Amico et al., 2019).

Además de los ejemplares de herbario, se sumó a la base de datos los ejemplares coleccionados a campo por los autores de este trabajo a lo largo de gran parte de la distribución geográfica de $L$. cuneifolia. Se recolectaron ejemplares a campo de la región Andes (Catamarca a Mendoza), región Central (San Luis y Córdoba) y de la región Este (Entre Ríos y Uruguay). Estos ejemplares se incorporaron a la colección de BCRU (Bariloche, Argentina). En base al registro de ejemplares de 


\section{G. C. Amico y R. Vidal-Russell - Hospedadores de Ligaria cuneifolia}

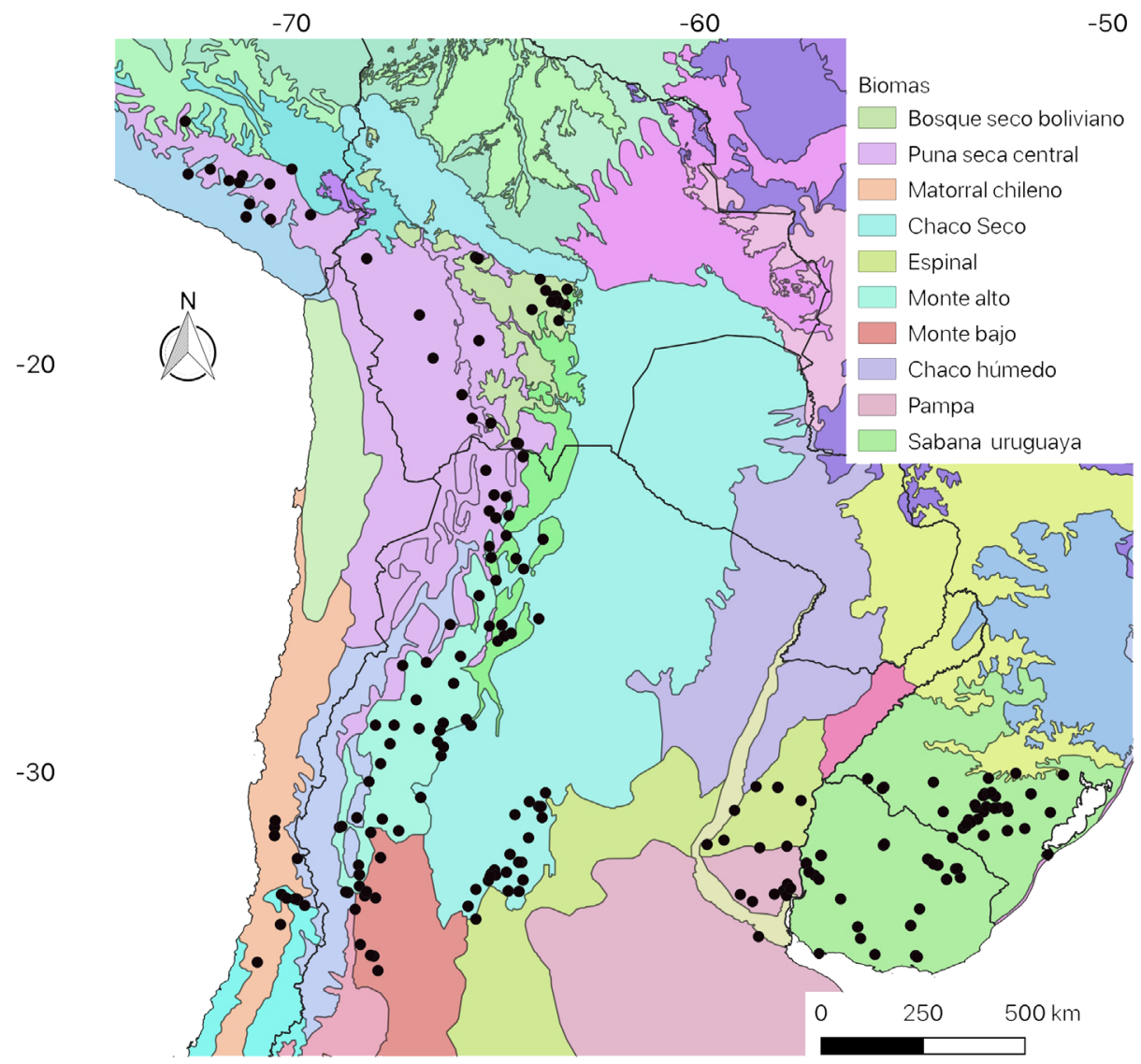

Fig. 1. Distribución geográfica de Ligaria cuneifolia. Se indican las ecoregiones según Olson et al. (2001).

herbarios y colecciones personales se construyó una matriz a nivel de especie, género y familia, para cada región geográfica de la distribución de $L$. cuneifolia. La nomenclatura de las especies sigue el Catálogo de Plantas Vasculares de la Argentina (Zuloaga et al., 2008).

Para medir la diversidad de hospedadores nativos utilizados por L. cuneifolia calculamos el estadístico $\mathrm{K}_{\mathrm{Q}}$ propuesto por Kavanagh \& Burns (2012). El valor $K_{Q}$ es equivalente a otras medidas de diversidad como por ejemplo, el índice de Shannon, incorporando información sobre las abundancias relativas y la riqueza de especies. Por lo tanto, este estadístico nos permite comparar con otras especies de muérdagos. El valor $\mathrm{K}_{\mathrm{Q}}$ se calculó utilizando la fórmula, $\mathrm{K}_{\mathrm{Q}}=\beta /(\alpha+1)$, donde $\beta$ es el número de hospedadores y $\alpha$ es la cantidad de registros. El estadístico $K_{Q}$ se calculó a nivel de género y especie de los hospedadores.

\section{Resultados}

Se encontraron 210 hospedadores representandos por 46 especies en los ejemplares de herbarios en toda la distribución geográfica de Ligaria cuneifolia (Apéndice). De todos los hospedadores registrados 35 especies corresponden a hospedadores nativos. Estas 35 especies están comprendidas en 23 géneros y 12 familias (Tabla 1). Los géneros con mayor frecuencia de uso (número de registros) fueron Schinus, Prosopis, Geoffroea, Larrea y Vachellia (Tabla 1); mientras que las familias fueron Fabaceae, Anacardiaceae y Zygophyllaceae. 
También se registraron más de 11 especies de hospedadores no nativos, principalmente de la familia Rosaceae (Apéndice).

Respecto a las regiones geográficas de distribución de L. cuneifolia (Andes, Centro y Este), la mayoría de los géneros y familias están compartidos entre las tres regiones geográficas (Tabla 1). La región Andes fue la que presentó una mayor diversidad de especies, género y familias de hospedadores. En esta región se registraron, 32 especies, comprendidas en 21 géneros y 11 familias.
Los géneros más frecuentemente utilizados en la región Andes fueron Prosopis, Schinus, Larrea, Geoffroea y Vachellia. La región Central fue la menos diversa, con sólo 5 géneros, siendo Geoffroea el género más frecuentemente parasitado. En la región Este, se registraron 7 géneros en total, siendo Schinus el más frecuentemente utilizado. El género Zanthoxylum de la familia Rutaceae fue único para esta región, pero sólo con un registro. El $\mathrm{K}_{\mathrm{Q}}$ de Ligaria cuneifolia para las especies nativas a nivel de especies fue de 0.23 y a nivel género de 0.14 .

Tabla 1. Géneros y familias de hospedadores usados por Ligaria cuneifolia en cada una de las regiones geográficas. Para cada género se indica el número de registros.

\begin{tabular}{|c|c|c|c|c|}
\hline Generos & Familia & Andes & Centro & Este \\
\hline Adesmia DC. & Fabaceae & 1 & & \\
\hline Atamisquea Miers & Capparaceae & 3 & & \\
\hline Bougainvillea Comm. ex Juss. & Nyctaginaceae & 2 & & \\
\hline Bredemeyera Willd. & Polygalaceae & 1 & & \\
\hline Bulnesia Gay & Zygophyllaceae & 3 & & \\
\hline Celtis L. & Cannabaceae & 1 & & 2 \\
\hline Condalia Cav. & Rhamnaceae & 3 & & \\
\hline Ephedra L. & Ephedraceae & 3 & & \\
\hline Geoffroea Jacq. & Fabaceae & 13 & 4 & 3 \\
\hline Larrea Ortega & Zygophyllaceae & 19 & & \\
\hline Lithraea Miers & Anacardiaceae & 2 & 1 & 4 \\
\hline Lycium L. & Solanaceae & 7 & & \\
\hline Porlieria Ruiz \& Pav. & Zygophyllaceae & 4 & & \\
\hline Prosopidastrum Burkart & Fabaceae & 1 & & \\
\hline Prosopis L. & Fabaceae & 34 & 2 & 1 \\
\hline Proustia Lag. & Asteraceae & 1 & & \\
\hline Schinopsis Engl. & Anacardiaceae & 2 & & \\
\hline Schinus L. & Anacardiaceae & 22 & 2 & 20 \\
\hline Trevoa Miers & Rhamnaceae & 1 & & \\
\hline Vachellia Wight \& Arn. & Fabaceae & 18 & 2 & 4 \\
\hline Zanthoxylum L. & Rutaceae & & & 1 \\
\hline Zuccagnia Cav. & Fabaceae & 4 & & \\
\hline Total & & 21 & 5 & 7 \\
\hline
\end{tabular}




\section{G. C. Amico y R. Vidal-Russell - Hospedadores de Ligaria cuneifolia}

\section{Discusión Y CONCLUSIÓN}

Ligaria cuneifolia es una especie generalista, parasitando a más de 30 especies nativas a lo largo de toda la distribución geográfica. El uso de hospedadores no estuvo directamente asociado a las áreas geográficas. La región Andes es donde L. cuneifolia parasita mayor diversidad de hospedadores, esto puede estar relacionado con que es la región que abarca más biomas. Mientras que la región Central que solamente abarca el bioma de Chaco seco es donde la diversidad de hospedadores es menor. Cuatro de los cinco géneros más frecuentemente utilizados (con más de 10 registros) Prosopis, Schinus, Geoffroea y Vachellia están presentes en las tres regiones geográficas. Sólo Larrea, con cerca 20 registros esta solamente presente en la región Andes. La mayoría de los registros sobre el género Prosopis son en la región Andes, en las otras dos regiones son utilizados pero en baja frecuencia.

Los géneros más parasitados, con más de 30 registros, fueron Schinus y Prosopis. Schinus es utilizado en casi la misma proporción en la región Este y Andes, mientras que Prosopis es más frecuentemente utilizado en la región Andes. Los géneros Prosopis y Schinus incluyen alrededor de 30 especies en Sudamérica (Zuloaga et al., 2008). Son géneros numerosos y si no se cuenta con partes reproductivas en el momento de la colección es difícil determinar la especie. El número de hospedadores a nivel de especie para estos dos géneros puede ser aun mayor a los reportados en este estudio.

El valor $\mathrm{K}_{\mathrm{Q}}$ de Ligaria cuneifolia encontrado en este trabajo, está dentro de los rangos reportados para el muérdagos generalistas en otras partes del mundo (Kavanagh \& Burns, 2012; Amico et al., 2019). Un estudio reciente (Amico et al., 2019) muestra que el uso del índice $\mathrm{K}_{\mathrm{Q}}$ está sujeto a sesgos según el tamaño de la muestra y es recomendable calcularlo a nivel de especie y género. Este estadístico podría mejorarse incorporando reglas de detención similares a las empleadas en los relevamientos a campo empleados en otros estudios con muérdagos (Watson et al., 2017).

Además de las especies acá reportadas, también L. cuneifolia ha sido registrada parasitando especies de cactáceas (Mauseth et al., 2015). Otra especie de Loranthaceae que también ha sido reportada parasitando cactus, es Tristerix aphyllus G.Don en el Matorral chileno. Tristerix aphyllus parasita solamente a este grupo de plantas, en especial a los géneros Echinopsis Zucc. and Eulychnia Phil. (Medel et al., 2002). Tristerix aphyllus es una apoespecie de T. corymbosus (L.) Kuijt, y se ha propuesto que se originó por un cambio del uso de hospedadores (Amico et al., 2007). Ligaria y Tristerix serían los únicos géneros dentro de Lorantáceas que parasitan a Cactáceas. Otro caso particular encontrado en Ligaria cuneifolia es el uso de Ephedra L. (Ephedraceae) como hospedador. Hasta la fecha no hay registro de muérdagos que utilicen como hospedadores a otros miembros de Gnetopsida.

A lo largo de su distribución Ligaria cuneifolia es simpátrica con otras especies de Lorantáceas cercanas filogenéticamente (Vidal-Russell \& Nickrent, 2008; Liu et al., 2018). En la región Andes y Central, L. cuneifolia es simpátrica con Tristerix verticillatus (Ruiz \& Pav.) Barlow \& Wiens, Tripodanthus flagellaris Tiegh. y Struthanthus spp. Mart.; mientras que en la región Este, L. cuneifolia es simpátrica con $T$. flagellaris y $T$. acutifolius (Amico et al., 2007, 2012; Amico \& Nickrent, 2009). Estas especies de muérdagos han sido observadas en el mismo área junto a L. cuneifolia, pero nunca compartiendo el mismo hospedador. En otras partes del mundo se ha reportado que no hay una superposición de uso de hospedadores entre especies simpátricas de muérdagos (Hawksworth \& Wiens, 1996; Jerome \& Ford, 2002; Fadini, 2011; Amico et al., 2019).

Además del uso de hospedadores nativos se ha registrado a $L$. cuneifolia parasitando especies no nativas que son frutales u ornamentales. En otras partes del mundo se han reportado impactos negativos de los muérdagos sobre la producción agrícola y forestal (Parker \& Riches, 1993; Mathiasen et al., 2008). En el caso de L. cuneifolia podría tener un efecto sobre cultivos de olivos (Olea) y frutas de carozo (Prunus). Es necesario realizar estudios a campo para determinar la intensidad de infección y los efectos negativos que puede tener sobre el hospedador en la producción de frutas.

En conclusion, $L$. cuneifolia es una especie de muérdago generalista, parasitando a numerosas especies de diferentes familias, siendo las familias Fabaceae, Anacardiaceae y Zygophyllaceae las más 
frecuentemente parasitadas. Dentro de las regiones geográfias, la región Andes fue la que presentó una mayor diversidad de especies, género y familias; mientras que la Región central fue la menos diversa. A pesar de presentar una distrubicuón disyunta, $L$. cuneifolia no tiene hospedadores específicos en cada una de las regiones geográficas.

\section{Contribución de AUtores}

Ambos autores, participaron en la realización de la investigación, interpretación de datos, preparación de la figura y redacción del manuscrito.

\section{Agradecimientos}

Agradecemos a los curadores de los diversos herbarios que fueron visitados por su asistencia en la recopilación de datos que se utilizaron en este estudio. También agradecemos a dos revisores anónimos que realizaron aportes para mejorar este trabajo. Los autores pertenecen al Consejo Nacional de investigación Científicas y Técnicas (CONICET). Para el desarrollo de este trabajo se contó con financiamiento de Fondo IBOL y PIP 11420110100085 .

\section{Bibliografía}

ABBIATTI, D. 1946. Las Lorantáceas Argentinas. Rev. Mus. La Plata, Sec. Bot. 7, 1-110.

AMICO, G.C. \& D.L. NICKRENT. 2009. First Report of the Mistletoe Tristerix verticillatus on Schinus fasciculata from the Sierra de San Luis, Argentina. Plant Dis. 93: 317.

https://doi.org/10.1094/PDIS-93-3-0317A

AMICO, G.C., D.L. NICKRENT \& R. VIDALRUSSELL. 2019. Macroscale Analysis of mistletoe host ranges in the Andean Patagonian Forest. Plant Biol.https://doi.org/10.1111/plb.12900

AMICO, G.C., R. VIDAL-RUSSELL, M.A. GARCIA \& D.L. NICKRENT. 2012. Evolutionary history of the South American mistletoe Tripodanthus (Loranthaceae) using nuclear and plastid markers. Syst. Bot. 37: 218-225. https://doi.org/10.1600/036364412X616783
AMICO, G.C., R. VIDAL-RUSSELL \& D.L. NICKRENT. 2007. Phylogenetic relationships and ecological speciation in the mistletoe Tristerix (Loranthaceae): the influence of pollinators, dispersers, and hosts. Am. J. Bot. 94: 558-567. https://doi.org/10.3732/ajb.94.4.558

BARLOW, B.A. 1983. Biogeography of Loranthaceae and Viscaceae. In: M. CALDER \& P. BERNHARDT (eds.) The Biology of Mistletoes, pp. 19-45. Academic Press.

DOWNEY, P.O. 1998. An inventory of host species for each aerial mistletoe species (Loranthaceae and Viscaceae) in Australia. Cunninghamia 5: 685-720.

FADINI, R.F. 2011. Non-overlap of hosts used by three congeneric and sympatric loranthaceous mistletoe species in an Amazonian savanna: host generalization to extreme specialization. Acta Bot. Bras. 25: 337-345. http://dx.doi.org/10.1590/ S0102-33062011000200010

GARCILLÁN, P.P. \& E. EZCURRA. 2011. Sampling procedures and species estimation: testing the effectiveness of herbarium data against vegetation sampling in an oceanic island. J. Veg. Sci. 22:273-280. https://doi.org/10.1111/j.1654-1103.2010.01247.x

HAWKSWORTH, F.G. \& D. WIENS. 1996. Dwarf Mistletoes: Biology, Pathology and Systematics, Agricultural Handbook No. 709. USDA Forest Service, Washington D.C.

JEROME, C.A. \& B.A. FORD. 2002. Comparative population structure and genetic diversity of Arceuthobium americanum (Viscaceae) and its Pinus host species: insight into host-parasite evolution in parasitic angiosperms. Mol. Ecol. 11: 407-420.

KAVANAGH, P.H. \& K.C. BURNS. 2012. Mistletoe macroecology: spatial patterns in species diversity and host use across Australia. Biol. J. Linn. Soc. 106: 459-468.

KUIJT, J. \& B. HANSEN. 2015. Loranthacceae. In: KUIJT J., HANSEN B. (eds.), The Families and Genera of Vascular Plants: Flowering Plants, Eudicots, pp 73-119. Springer, Berlin, Germany.

KUIJT, J.1990. A second species of Ligaria (Loranthaceae). Brittonia 42: 66-69.

LIRA-NORIEGA, A. \& A.T. PETERSON. 2014. Rangewide ecological niche comparisons of parasite, hosts and dispersers in a vector-borne plant parasite system. J. Biog. 41: 1664-1673. https://doi. org/10.1111/jbi.12302.

LIU, B., C. LEA, R. BARRETTE, D.L. NICKRENT, Z. CHENA, L. LUA, L. \& R. VIDAL-RUSSELL. 


\section{G. C. Amico y R. Vidal-Russell - Hospedadores de Ligaria cuneifolia}

2018. Historical biogeography of Loranthaceae (Santalales): Diversification agrees with emergence of tropical forests and radiation of songbirds. Mol. Phyl. Evol 124: 199-212. https://doi.org/10.1016/j. ympev.2018.03.010

MATHIASEN, R.L., D.L. NICKRENT, D.C. SHAW, D.M. \& WATSON. 2008. Mistletoes: pathology, systematics, ecology, and management. Plant Dis. 92: 988-1006. https://doi.org/10.1094/PDIS-92-70988

MAUSETH, J.D., S. BENIGNO, F.C. HUAMANI \& C. OSTOLAZA. 2015. Host response and endophyte structure of the mistletoe Ligaria cuneifolia (Loranthaceae) parasitizing Corryocactus brevistylus (Cactaceae). Haseltonia 21: 3-13. https://doi.org/10.2985/026.021.0103

MEDEL, R., C. BOTTO-MAHAN. C. SMITHRAMIREZ, M.A. MENDEZ, C.G. OSSA, L.N. CAPUTO, W.L. GONZALES. 2002. Historia natural cuantitativa de una relación parásitohospedero: el sistema Tristerix-cactáceas en Chile semiárido. Rev. Chil. Hist. Nat. 75: 127-140.

NORTON, D.A. \& M.A. CARPENTER. 1998. Mistletoes as parasites: host specificity and speciation. Trends Ecol. Evol. 13: 101-105.

NORTON, D.A. \& P.J. DE LANGE.1999. Host specificity in parasitic mistletoes (Loranthaceae) in New Zealand. Funct. Ecol. 13: 552-559.
OLSON, D.M., E. DINERSTEIN, E.D. WIKRAMANAYAKE, N.D. BURGESS, G.V.N. POWELL, E.C. UNDERWOOD, J.A. D'AMICO, I. ITOUA, H.E. STRAND, J.C. MORRISON, C.J. LOUCKS, T.F ALLNUTT, T.H. RICKETTS, Y. KURA, J.F. LAMOREUX, W.W. WETTENGEL, P. HEDAO \& K.R. KASSEM. 2001. Terrestrial Ecoregions of the World: A New Map of Life on Earth: A new global map of terrestrial ecoregions provides an innovative tool for conserving biodiversity. BioScience 51: 933-938. https://doi.org/10.1641/00063568(2001)051[0933:TEOTWA]2.0.CO;2

PARKER, C. \& C.R. RICHES. 1993. Parasitic weeds of the world: biology and control. CAB international.

VIDAL-RUSSELL, R. \& D.L. NICKRENT. 2008. Evolutionary relationships in the showy mistletoe family (Loranthaceae). Am. J. Bot. 95: 1015-1029. https://doi.org/10.3732/ajb.0800085

WATSON, D.M., K.V. MILNER \& A. LEIGH. 2017. Novel application of species richness estimators to predict the host range of parasites. Int. J Parasitol. 47: 31-39. https://doi.org/10.1016/j.ijpara.2016.10.001

ZULOAGA, F.O., O. MORRONE \& M.J. BELGRANO. 2008. Catálogo de las plantas vasculares del Cono Sur (Argentina, southern Brazil, Chile, Paraguay and Uruguay). Volume 2: Dicotyledoneae: Acanthaceae-Fabaceae (Abarema-Schizolobium). Missouri Botanical Garden Press, Saint Louis. 
Apéndice. Especies nativas y no nativas parasitadas por Ligaria cuneifolia a la largo de su distribución geográfica en las regiones Andes, Este y Centro de Sudamérica. Los números indican la cantidad

de veces que se registró parasitismo en cada especie. Para las especies no nativas se indica el uso, ornamental o frutícola.

\begin{tabular}{|c|c|c|c|c|c|}
\hline Especie & Autor & Familia & Andes & Centro & Este \\
\hline \multicolumn{6}{|l|}{ Especies nativas } \\
\hline Lithraea sp. & Miers. & Anacardiaceae & 1 & & \\
\hline Lithraea brasiliensis & Marchand & Anacardiaceae & & & 4 \\
\hline Lithraea molleoides & (Vell.) Engl. & Anacardiaceae & 1 & 1 & \\
\hline Schinopsis marginata & Engl. & Anacardiaceae & 2 & & \\
\hline Schinus sp. & $\mathrm{L}$. & Anacardiaceae & 17 & & 1 \\
\hline Schinus dependens & Ortega & Anacardiaceae & 1 & & 2 \\
\hline Schinus fasciculata & (Griseb.) I.M. Johnst. & Anacardiaceae & 1 & 2 & 2 \\
\hline Schinus longifolius & Speg. & Anacardiaceae & & & 7 \\
\hline Schinus molle & L. & Anacardiaceae & 2 & & 6 \\
\hline Schinus polygama & (Cav.) Cabrera & Anacardiaceae & 1 & & 2 \\
\hline Proustia sp. & Lag. & Asteraceae & 1 & & \\
\hline Celtis sp. & L. & Cannabaceae & 1 & & \\
\hline Celtis tala & Gillies ex Planch. & Cannabaceae & & & 2 \\
\hline Atamisquea emarginata & Miers ex Hook. \& Arn. & Capparaceae & 3 & & \\
\hline Ephedra sp. & L. & Ephedraceae & 2 & & \\
\hline Ephedra breana & Phil. & Ephedraceae & 1 & & \\
\hline Adesmia sp. & DC. & Fabaceae & 1 & & \\
\hline Geoffroea decorticans & (Gillies ex Hook. \& Arn.) Burkart & Fabaceae & 13 & 4 & 3 \\
\hline Prosopidastrum globosum & (Gillies ex Hook. \& Arn.) Burkart & Fabaceae & 1 & & \\
\hline Prosopis sp. & L. & Fabaceae & 25 & 2 & \\
\hline Prosopis alba & Griseb. & Fabaceae & 1 & & \\
\hline Prosopis chilensis & Stuntz & Fabaceae & 1 & & \\
\hline Prosopis flexuosa & DC. & Fabaceae & 2 & & \\
\hline Prosopis juliflora & DC. & Fabaceae & 1 & & \\
\hline Prosopis kuntzei & Harms \& Hassl. & Fabaceae & 2 & & \\
\hline Prosopis nigra & Hieron. & Fabaceae & 2 & & 1 \\
\hline Vachelia caven & (Molina) Seigler \& Ebinger & Fabaceae & 11 & & 2 \\
\hline Vachelia sp. & Wight \& Arn. & Fabaceae & 7 & 2 & 2 \\
\hline Zuccagnia punctata & Cav. & Fabaceae & 4 & & \\
\hline Bougainvillea spinosa & (Cav. Heimerl & Nyctaginaceae & 2 & & \\
\hline Bredemeyera colletioides & (Phil.) Chodat & Polygalaceae & 1 & & \\
\hline Condalia microphylla & Cav. & Rhamnaceae & 3 & & \\
\hline Trevoa quinquenervia & Gillies \& Hook. & Rhamnaceae & 1 & & \\
\hline
\end{tabular}




\section{G. C. Amico y R. Vidal-Russell - Hospedadores de Ligaria cuneifolia}

\begin{tabular}{|c|c|c|c|c|c|c|}
\hline Especie & Autor & Familia & Andes & Centro & Este & \\
\hline Zanthoxylum fagara & Sarg. & Rutaceae & & & 1 & \\
\hline Lycium sp. & L. & Solanaceae & 6 & & & \\
\hline Lycium tenuispinosum & Miers (GCI) & Solanaceae & 1 & & & \\
\hline Bulnesia retama & (Gillies ex Hook. \& Arn.)Griseb. & Zygophyllaceae & 3 & & & \\
\hline Larrea sp. & Cav. & Zygophyllaceae & 5 & & & \\
\hline Larrea divaricata & Cav. & Zygophyllaceae & 12 & & & \\
\hline Larrea nitida & Cav. & Zygophyllaceae & 2 & & & \\
\hline Porlieria chilensis & I.M.Johnst. & Zygophyllaceae & 4 & & & \\
\hline Especies no nativas & & & & & & Uso \\
\hline Nerium sp. & $\mathrm{L}$. & Apocynaceae & 1 & & & Ornamental \\
\hline Robinia pseudoacacia & L. & Fabaceae & 1 & & & Ornamental \\
\hline Tipuana sp. & Benth. & Fabaceae & 1 & & & Ornamental \\
\hline Punica granatum & $\mathrm{L}$. & Lythraceae & 1 & & & Frutícola \\
\hline Olea europea & $\mathrm{L}$. & Oleaceae & 3 & & & Frutícola \\
\hline Crataegus sp. & L. & Rosaceae & 1 & & & Ornamental \\
\hline Malus domestica & (Suckow) Borkh. & Rosaceae & 2 & & & Frutícola \\
\hline Prunus sp. & $\mathrm{L}$. & Rosaceae & 3 & & & Frutícola \\
\hline Prunus armeniaca & Blanco & Rosaceae & 1 & & & Frutícola \\
\hline Prunus dulcis & D.A.Webb & Rosaceae & 1 & & & Frutícola \\
\hline Prunus persica & (L.) Batsch & Rosaceae & & & 1 & Frutícola \\
\hline Pyrus communis & $\mathrm{L}$. & Rosaceae & 3 & & & Frutícola \\
\hline
\end{tabular}


